\title{
Helmuth Feilke
}

\section{Kontext - Zeichen - Kompetenz \\ Wortverbindungen unter sprachtheoretischem Aspekt}

\begin{abstract}
Die kontinuierliche Ausweitung des Gegenstandsbereichs der Phraseologie in den vergangenen 30 Jahren geht einher mit einer Pragmatisierung theoretischer Grundannahmen in der Disziplin selbst. Damit ist nicht die Frage der „,Verwendung von Phraseologismen“ gemeint, sondern das zunehmende Gewicht der Frage, welche Ausdrücke Sprecherinnen und Sprecher pragmatisch als Einheiten der Sprachproduktion und des Sprachverstehens behandeln. Der negativ bestimmten, gut operationalisierbaren Eingrenzung des Phraseologischen als eines Ausdrucksbereichs, der grammatisch und semantisch postulierten Wohlgeformtheitsbedingungen nicht genügt, korrespondiert fachgeschichtlich die positive Bestimmung als Tradition des Sprechens. Rekurrente Ausdrucksselektion und -kombination kann diachron durch semantischen, grammatischen und phonologischen Strukturverlust geprägt sein, aber dieser ist nicht die Ursache, sondern eine mögliche Folge idiomatischer Prägung. Die Ursache ist die durch koordinierte Selektivität der Sprecherinnen und Sprecher etablierte, konventionell-arbiträre Einschränkung der Produktions- und Interpretationsoptionen. Kommunikations- und sprachtheoretisch gibt es deshalb gute Gründe, einen weiten Bereich der Bildung usueller komplexer Ausdrücke ohne Strukturverlust anzunehmen. Er umfasst die pragmatisch konstituierten Leistungseinheiten der idiomatischen Kompetenz. Der so genannte „feste“ Bereich sprachlichen Ausdrucks besteht vor allem darin, dass Sprecherinnen und Sprecher die inhaltsseitig kontextuell indizierten und ausdrucksseitig als typisch bewerteten usuellen Selektionen und Kombinationen ihres Idioms kennen. Die Aktualität dieser Fragen spiegelt sich im Kontextualisierungsparadigma der jüngeren Pragmatik, das Kommunikation wesentlich als ein pars-prototo-Geschehen versteht, in der „Kollokations-Konjunktur“ in Lexikologie und -graphie, die es nahe legt, Wortbedeutung pragmatisch als Bedeutung text- und domänengebundener Kollokationen zu behandeln und ebenso in der Grammatikalisierungsdiskussion, die systematisch mit Einheiten zwischen Lexikon und Grammatik rechnet.

Die problemgeschichtlich orientierte Darstellung rekonstruiert eine konvergente Entwicklung im Verhältnis von phraseologischer Theorie und allgemeiner Sprachtheorie.
\end{abstract}

\section{Problemgeschichte und Theoriewandel}

Theoriebildung und Problemgeschichte bilden in der Sprachwissenschaft stets einen engen Zusammenhang. Dabei ist der Theoriewandel nicht nur Ursache, sondern auch Folge einer im Fachdiskurs kontinuierlich veränderten 
Problemsicht und Problemqualität. Statt die für das Thema einschlägigen Theoriefragen aus der Erkenntnistheorie, der Kommunikationstheorie und schließlich der Sprachtheorie abzuleiten, entwickelt der folgende Beitrag die theoretisch relevanten Fragen und Antworten aus einer problemgeschichtlichen Darstellung des Diskurses der Phraseologie in den vergangenen 30 Jahren. Die Untersuchung der Gegenstandsverschiebungen in diesem Zeitraum öffnet den Blick für den Theoriewandel und seine Ursachen und erleichtert die Eingrenzung der aktuell anstehenden theoretischen Probleme.

Titel haben programmatischen Charakter. Ein Blick auf die Titel der IDSJahrestagungen zur Phraseologie selbst ist deshalb schon instruktiv: Der Titel der Jahrestagung 1988 zum Thema bediente sich noch einer bewährten linguistischen Zwillingsformel: „Wortbildung und Phraseologie“. Beide Konzepte sind etabliert und wer etablierte Konzepte unter dem Signum einer Zwillingsformel zusammenführt, der ist zugleich innovativ und - qua Formel - auf der sicheren Seite. Ganz anders sieht es im Jahr 2003 mit dem Titel aus: „Den Nagel auf den Kopf treffen - Wortverbindungen: mehr oder weniger fest." Ich schlage folgende Lesart vor: Der diesjährige Titel inszeniert - gewissermaßen exemplarisch - ein phraseologisches Rollenspiel und thematisiert damit implizit einen Rollenwandel der Phraseologie selbst. Ein klassischer Phraseologismus gibt noch den Haupttitel ab: „Den Nagel auf den Kopf treffen", aber er wird ausdrücklich als bloßes Phraseologiezitat ohne eigentliche Referenz markiert. Der Untertitel öffnet dann ein weites Feld: „Wortverbindungen - mehr oder weniger fest". Was in der Sprache ist keine Wortverbindung und was ist nicht mehr oder weniger fest? Der Untertitel reinterpretiert (nicht reliteralisiert) die semantisch figurierte Wortverbindung mehr oder weniger und liefert damit selbst ein Beispiel für die gegenwärtig in der Forschung so prominente „Rollen-Flexibilität“ des vermeintlich Fixierten. Schließlich: Dem Tagungstitel liegt insgesamt ein Formulierungsschema oder -modell, ein Rollenmuster mit zwei syntaktisch unabhängigen, aber textlich kooperierenden Mitspielern zugrunde: phraseologisch metaphorischer Haupttitel plus literal referentieller Untertitel. Das Ausdrucksschema ist nur transphrastisch und im Hinblick auf die Kategorie Text analysierbar. Es liegt schon auf der Grenze dessen, was sinnvoll noch als Wort-Verbindung verstanden werden kann. Gleichwohl steht die idiomatische Prägung dieses gerade auch bei wissenschaftlichen Überschriften produktiven Schemas außer Frage. Man kennt es, und es gehört zur idiomatischen Kompetenz, zu wissen, in welchem Kontext es funktioniert.

Der Seitenblick auf die Überschrift der Tagung, die vom Spiel mit einem Phraseologismus übergeht zu einer mehr oder weniger freien Wortverbindung bis hin zu einer domänen- und funktionstypischen Strukturformel, die selbst nur noch eine Text-, aber keine Wortbindung mehr aufweist, führt zur Leitthese meines Beitrags, die ich mit Pragmatisierung der Phraseologie überschreibe: 
Die seit Beginn der 70er Jahre intensivierte Erforschung der Phraseologie ist gekennzeichnet durch eine in drei Hauptstufen verlaufende Pragmatisierung der Theorie, die zugleich zu einer verstärkten Integration phraseologischer Fragestellungen in die allgemeine Sprachtheorie führt. Pragmatisierung soll heißen, dass das Spektrum idiomatischer Prägung extensional nicht mehr über strukturlinguistisch zu fassende Irregularitäten, sondern zunehmend über das Kriterium der pragmatisch usuellen semiotischen Einheiten des Sprachgebrauchs definiert wird. In dem Maße, in dem im Zuge dieser Entwicklung der Quasi-Lexemstatus idiomatischer Prägungen zurückgenommen und relativiert wird, ändert sich der Gegenstandsbereich der Phraseologie, aber auch grundlegende Konzepte der Sprachtheorie. Für die Definition sprachlicher Zeichenhaftigkeit selbst sind genuin pragmatische Gesichtspunkte zu berücksichtigen.

Im Wesentlichen beziehe ich mich in der folgenden Darstellung auf die Diskussion zum deutschen Sprachbereich, wobei der Wandel in allen Philologien ähnlich verläuft. Wenn ich von einer Pragmatisierung in drei Hauptstufen spreche, so sind damit in aller Kürze folgende Etappen gemeint: Ich unterscheide die Phase einer vorpragmatischen Idiomatik, die bis Ende der 70er Jahre andauert, eine zweite, die die Diskussion in den 80er Jahren bestimmt und die ich unter die Überschrift „Pragmatisierung der Idiomatik“ stelle. Schließlich kann mit Beginn der 90er Jahre ein erneuter Wandel und Perspektivwechsel festgestellt werden, dessen Konsequenzen auch die gegenwärtige Diskussion prägen. Ich fasse ihn unter dem Gliederungspunkt „Konstruktive Pragmatik“ und werde in diesem Kapitel einige der virulenten Probleme genauer in den Blick nehmen.

\section{Vorpragmatische Idiomatik}

Die vorpragmatische Idiomatik ist die Phase der Konsolidierung der Phraseologie, der Herausarbeitung der zentralen Bestimmungsmerkmale der Polylexikalität, Festigkeit und Figuriertheit bzw. Idiomatizität auf der Grundlage von Vorarbeiten vor allem auch aus der sowjetischen Phraseologie. Den Stand repräsentieren die Überblicksarbeiten z. B. von Burger et al. (1982), Fleischer (1982), Greciano (1983). Kernpunkt der Begriffsbildung ist die Analogie von Wort und idiomatisch geprägtem Ausdruck. Die Aufmerksamkeit liegt auf den Eigenschaften, die Phraseologismen strukturell mit Wörtern teilen: transformationelle bzw. syntagmatische und paradigmatische Fixierung und nicht-kompositionelle Bedeutungskonstitution, syntaktische und semantische Irregularität also. Die folgenden Beispiele aus Müller (1997, S. 20) verdeutlichen diesen Gesichtspunkt. Bei den Beispielen zu a) verhalten sich die Binominale flexivisch kongruent wie komplexe Wörter, wobei nur das Zweitglied flektiert wird. Die Beispiele zu b) illustrieren das für den Wortbegriff zentrale Einschubverbot. 
a)

- $\quad$ ein [fix(*es) und fertiges] Buch

- $\quad \operatorname{der}\left[f i x\left({ }^{*} e\right)\right.$ und fertige] Konrad

- $\quad$ eine [klipp $\left({ }^{*} e\right)$ und klare] Stellungnahme

b)

- bei [Nacht und (*dickem) Nebel]

- [sage und (*wenn du willst) schreibe]

- [klipp und (*ziemlich) klar]

Das Lexikon gilt entsprechend einem Diktum Bloomfields als ,list of basic irregularities" (Bloomfield 1933, S. 274). Phraseologismen und Idiome werden wegen ihrer Metaphorizität bzw. Figuriertheit und ihrer literalen Synonyme im Lexikon funktional im Wesentlichen als sekundäre Nominationen mit vorwiegend emotiver Funktion analysiert (Fleischer 1982). Psycholinguistisch stellt man sich ihre Prozessierung als Übersetzungsvorgang vor, in dem die figurierte Gesamtbedeutung jedes $\mathrm{Mal}$ in der Interpretation reliteralisiert wird (vgl. z. B. Weinreich 1969/1980). In einer Zeit, in der die linguistische Kreativität im Sinne der generativen Grammatik das Zentralproblem der Theoriebildung ist, macht die Selbstdefinition der Phraseologie diese zu einer sprachtheoretisch randständigen Disziplin.

Die Arbeitsdefinition des Phraseologischen ist von Anfang an von Problemen geprägt. So ist die Abgrenzung nach unten zur Morphematik hin willkürlich. Denn auch Morpheme sind idiomatisch (schon Hockett 1956). Noch größere Probleme bereitet der Umstand, dass es weit verbreitet Festigkeit ohne Idiomatizität gibt, ebenso das schon immer mit behandelte Sprichwort, das eigentlich ein Mikrotext und satzsemantisch transparent und kompositionell ist. Gleichwohl sind Darstellungen der Phraseologie in dieser Phase dominiert von einem Leitbild strukturell disjunkter Klassen, wie es das folgende an Pilz (1978) orientierte Schema zeigt.

\section{Klassen-Modelle:}

Beispiel nach Pilz (1978)

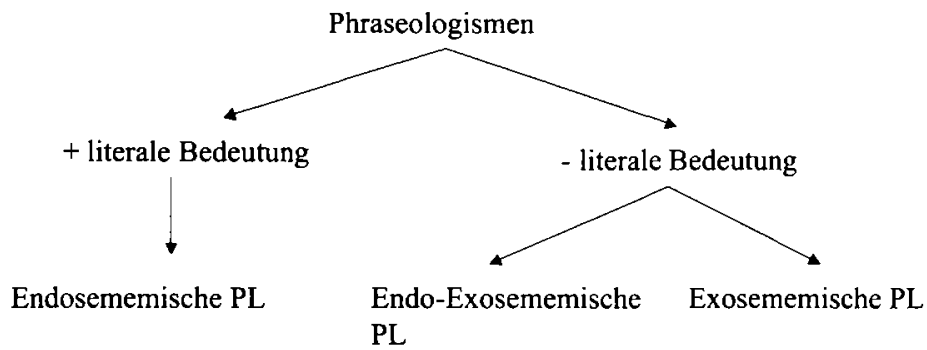

Dank sagen

Feuer fangen (sich

Bauklötze staunen begeistern) 


\section{Pragmatisierung der Idiomatik}

Die Phase der Pragmatisierung setzt ein mit der Untersuchung so genannter pragmatischer Idiome (bereits Burger 1973 im Anschluss an Makkai 1972). Sie ist aber nicht einfach eine deskriptive Erweiterung des Spektrums, sondern stellt gleich in mehrfacher Hinsicht die bis dahin gepflegte Grundbegrifflichkeit in Frage: Es geht bei den pragmatischen Idiomen nicht um Irregularität, sondern, ganz im Gegenteil, um eine pragmatische Regelhaftigkeit. Zwar steht bei einem Ausdruck wie „Guten Morgen!“ am Anfang noch die für den phraseologischen Charakter vermeintlich verantwortliche strukturelle Irregularität im Vordergrund des Interesses, aber die Umkehrung der Perspektive ist nicht mehr aufzuhalten, und so stellt etwa Thun (1978) im Blick auf den Ausdruck Das schlägt dem Fass den Boden aus! unabhängig von der ausdrucksinternen idiomatischen Qualität dieses Ausdrucks eine „äußere Fixiertheit“ (ebd. S. 242) fest. Der Ausdruck ist keine Nomination, er ist syntaktisch nicht einfach polylexikal, sondern ein Satz, und er hat vor allem und in erster Linie Äußerungswert. Florian Coulmas' (1981a) „Routine im Gespräch" kann als ein erster Höhepunkt der Entwicklung aufgefasst werden. Die Routineformel kann - und hierin gleicht sie dem Sprichwort semantisch und syntaktisch ausdrucksintern vollkommen regulär sein. Entscheidend ist die konventionelle Bindung an einen pragmatisch signifikanten Kontext. Versuche, die Routineformel - etwa unter dem Terminus „Satzlexem" - noch für das alte Paradigma zu retten, wirken schon Anfang der 80er Jahre anachronistisch. Die Versuche den Gesamtbereich zu fassen, führen zur Modellierung verschiedener Kontinuen des Idiomatischen, wie sie das folgende Schema zusammenfassend zeigt. In aller Regel wird dabei das Kontinuum über ein zentrales explanatives Merkmal modelliert, etwa den Grad der angenommenen Automatisierung (z. B. Bolinger 1976) oder den Grad der Ritualität (Lüger 1980). Übergreifend wird darin der Versuch deutlich, Gebrauchsmerkmale zu definierenden Merkmalen für „Festigkeit“ zu erklären.

Kontinuum -Modelle :

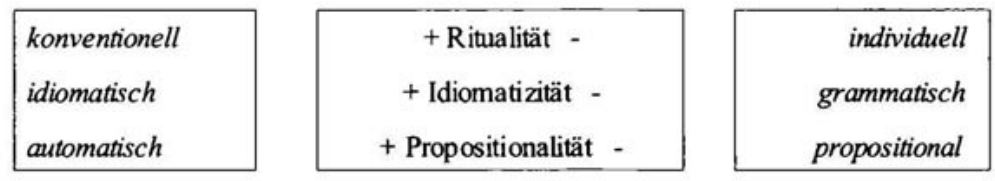

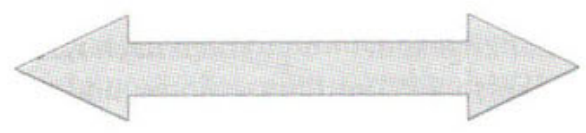

z. B. Bolinger (1976), Lüger (1980), Coulmas (1981b) 
Florian Coulmas (1981a) etabliert neben den bekannten phraseologischen Kriterien das Kriterium der zunächst nur auf Situationen bezogenen Rekurrenz des Gebrauchs. Weil Routineformeln etwa in sogenannten adjacency pairs (z. B. Entschuldigen Sie bitte! - Ach, das kann doch jedem passieren!) obligatorisch über den Satz hinaus pragmatisch gebunden sein können, führt die Perspektive konsequenterweise schon bald dazu, dass die syntagmatische Ausdehnung der in dieser Weise gebundenen sprachlichen Formen theoretisch kaum noch eine Rolle spielt. Das Kriterium der Polylexikalität als definitorisches Kriterium der Phraseologie kann sowohl nach unten hin (zum Beispiel: Hallo!) wie nach oben zu Satz und Text hin das Spektrum idiomatischer Prägung nicht begrenzen (vgl. auch Antos 1987, Stein 1995, Gülich 1997).

Nun könnte man die Routineformeln als einen Spezial- und Randbereich der Phraseologie abtun und in der Tat erscheinen sie in den ab Mitte der 80er Jahre verbreiteten Zentrum-Peripherie-Modellen auch genau dort, wie das folgende an Gläser (1986) orientierte Schema zeigt. Der Gesamtbereich wird erfasst, aber fraglos bildet hier noch der phraseologische „Kern“ das Zentrum.

\section{Zentrum-Peripherie-Modelle:}

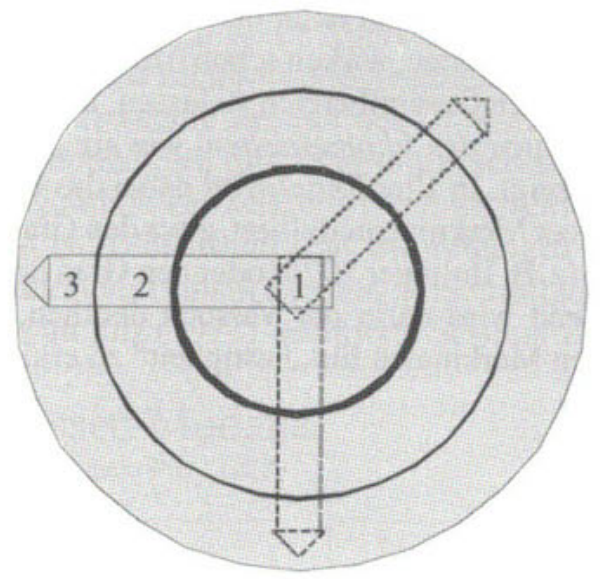

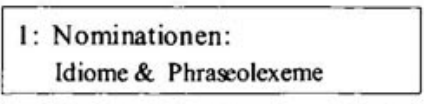

2: Reduzierte Propositionen Redewendungen, Binominale etc.

3: Propositionen

Routineformeln, Sprichwörter etc.

Beispiel nach Gläser (1986)

z.B. auch Fleischer (1983), Coulmas (1985), Hessky (1987), Dobrovols'kij (1995)

Die mit der Integration der Routineformeln aufgeworfenen theoretischen Probleme werden weiter zugespitzt und wirken nach meiner Einschätzung in einer fundamentalen Weise auf die Phraseologie und auch auf die Sprachtheorie zurück. Ausschlaggebend dafür sind zwei Argumente.

Erstes Argument: Das Kriterium der situativen Rekurrenz, wie es noch für Begrüßungsformeln, Entschuldigungen usw. gilt, wird - meines Wissens zu- 
erst von Hans Schemann (1989) - abstrahiert zum Kriterium einer fixierten rekurrenten Kontextkonstellation, die außer der Gesamtheit außersprachlicher auch die textlichen und sprachlichen Kontextparameter selbst betreffen kann. Die Bindung kann über die Situationsparameter und Handlungsrollen (z. B. Entschuldigungsformeln), sie kann aber auch inhaltlich über das Thema (z.B. Nominationsstereotype, linguistische Stereotype) oder einen sprachlichen Kontextparameter wie die Textsorte (Überschriften, funktionstypische lexikalische Kollokationen) hergestellt sein. Die pragmatische Fixierung innerhalb arbiträr und konventionell festgelegter Kontexte wird dabei nicht als ein zusätzliches, sondern als das entscheidende Kriterium etabliert. Schemann (1989, S. 1020) fordert, systematisch die Frage zu stellen,

„inwieweit die Fixierung auf irgendeiner Kontextebene - oder mehreren dieser Ebenen - bei Variabilität/Freiheit der anderen das allgemeinsprachliche Wesensmerkmal der phraseologischen Verbindung in all ihren Erscheinungsformen ist." (Schemann 1989, S. 1020; Hervorhebung H.F.)

Mit dieser Formulierung ist meines Erachtens von der Theorieentwicklung innerhalb der Phraseologie her bereits die Quintessenz der Pragmatisierung angesprochen. Wortverbindungen werden fest durch den Gebrauch und sind pragmatisch fixiert innerhalb konventionaler Gebrauchskonstellationen. Ein differenziertes Konzept solcher „Bindungsebenen“ im Sinne Schemanns entwickelt auch Wolfgang Schindler (1996).

Zweites Argument: Die Hervorhebung in dem Schemann-Zitat betont den nach meiner Auffassung wesentlichen Aspekt. Eine performative oder eine thematische Fixierung impliziert nicht notwendigerweise auch eine syntaktische oder semantische Fixierung. Eine Formel wie Ich liebe dich oder ein Binominal wie Kaffee und Kuchen ist nicht in erster Linie idiomatisch, weil wir hier bestimmte syntaktische und semantische Restriktionen haben (keine Passivtransformation, beschränkte Erweiterbarkeit, Irreversibilität). Sie sind zunächst in dem Sinne pragmatisch gebunden, dass sie die Kenntnis des üblichen Gebrauchszusammenhangs - etwa einer Liebeserklärung oder des nominativen Bezugs auf eine kulturell einschlägige Szenerie des Kaffeetrinkens - verlangen. Die Bezeichnungsleistung des Binominals „Kaffee und Kuchen" ist konventionell auf diese Szene bezogen. Es indiziert als sprachlicher ,frame“ konventionell eine bestimmte „scene“, um mit Charles Fillmore zu sprechen (vgl. hierzu Lambrecht 1984). Diese Bindung aber ist zu allererst eine pragmatische, eine Obligation des Ausdrucksgebrauchs. Formen struktureller Irregularisierung sind nicht nur diachron nachgeordnet, sie sind auch funktional nachrangig gegenüber dem Faktum der pragmatischen Regularisierung der Beziehung zwischen Kontextparametern und Ausdrucksformen. Klar formuliert Igor Mel'čuk (1998, S. 29) diesen Punkt: 
"All ready-made expressions, even if they are wholly compositional semantically and syntactically, are pragmatemes: they are non-compositional pragmatically."

(Mel'čuk 1998, S. 29, Hervorhebung H.F)

Diese Einsicht führt zwingend zu einer sowohl für die Phraseologie als auch für die Sprachtheorie sehr weitreichenden Konsequenz: Sprachliche Ausdrücke können im angesprochenen Sinne zugleich idiomatische Zeichen und syntaktisch und semantisch wohlgeformt sein. Die idiomatische Qualität solcher Ausdrücke als Zeichen ist pragmatisch entkoppelt und funktioniert pragmatisch entkoppelt von Eigenschaften ihrer syntaktischen und semantischen Struktur. Damit muss angenommen werden, dass es in jeder Sprache eine unbekannte Zahl pragmatisch konventioneller und zugleich strukturell motivierter, semantisch kompositioneller, komplexer Zeichen gibt. Dies können Wörter (Komposita), Phrasen und Sätze sein.

Ein meines Erachtens tragfähiges semiotisches Erklärungsmodell für diese Tatsache ist das auf Roland Barthes (1970) zurückgehende Modell der doppelten Signifikation. Der Bezug auf Barthes ist nicht zwingend. Gleichermaßen tragfähig - und begrifflich analog zum Konzept der doppelten Signifikation- wäre auch Hjelmslevs Konzept der Konnotation, speziell in der Interpretation durch Utz Maas (1985). Ich nutze das Schema der doppelten Signifikation hier, um zwei Ebenen der Idiomatizität zu unterscheiden.

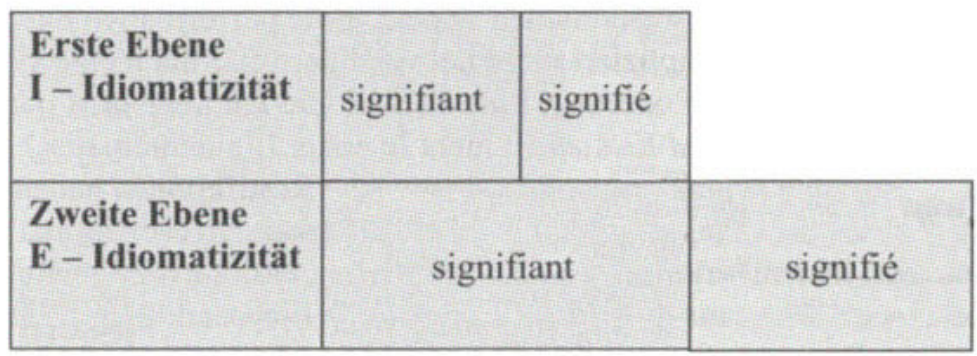

Doppelte Signifikation nach Barthes (1970)

Die primäre semiotische Relation ist die arbiträre und konventionelle und manchmal auch relativ motivierte Zuordnung von signifiant und signifié. Der typische Fall dafür ist das Wortzeichen. Aber auch Phraseologismen sind in diesem Sinne intern idiomatisch. Das Zeichen als Einheit von signifiant und signifié auf der ersten Ebene ist einerseits Mittel der Signifikation. Es kann aber - und das gilt insbesondere eben auch für strukturell motivierte und semantisch kompositionelle Zeichenkombinationen - andererseits als 
Folge durch den Gebrauch stets auch zum Zeichen des Gebrauchszusammenhangs selbst werden. Hier wird auf der zweiten Signifikationsebene ein komplexes signifiant - ein Redezeichen - pragmatisch durch den Gebrauch geprägt. Man kann mit Maas (1985) auch sagen: Es wird konnotativ geprägt.

Eine Sentenz wie Wer zu spät kommt, den bestraft das Leben ist auf der Ebene ihrer syntaktischen und semantischen Struktur absolut regulär und auch semantisch kompositionell. Auf der zweiten Signifikationsebene aber, die durch den Gebrauch die Lesart und die möglichen Kontextsituationen des erneuten Gebrauchs einschränkt, ist sie idiomatisch. Sie ist idiomatisch in dem Sinne, dass sie verbindlich ein spezifisches Verwendungsschema konnotiert. Der Gebrauch der Formel ist nicht passend um jemanden zu begrüßen, der zu spät zu einer Verabredung erscheint. Diese Zusammenhänge hat bereits Peter Grzybek in seinen Analysen zum Sprichwort im Sinne der Definition Mel'čuks und unter Rückgriff auf das Schema der doppelten Signifikation herausgestellt (vgl. Grzybek 1984a, b).

Schemanns Konzept der variablen Fixierung alternativer Kontexte und Mel'čuks Definition des Pragmatems verdeutlichen die Option der Entkopplung innerer - strukturlinguistisch fassbarer - und äußerer - nur pragmatisch . zu bestimmender - Kriterien für idiomatische Prägung.

Dies bedeutet in der Konsequenz, dass semantisch und syntaktisch wohlgeformte Ausdrücke ohne jede Einschränkung idiomatisch sein können, wenn sie hinsichtlich eines oder mehrerer pragmatischer Kontextparameter geprägt und das heißt, bereits interpretiert sind. Die syntaktisch-semantische Konstituenz des Ausdrucks ist nicht die Ursache seiner idiomatischen Prägung. Ausdrücke, die intern nicht strukturiert sind (z. B. Hallo!), können in gleicher Weise im Sinne der E-Idiomatizität idiomatisch geprägt sein wie Ausdrücke mit syntaktisch regulärer und semantisch kompositioneller Struktur (Gut, dass ich Sie treffe; Eine Schwalbe macht noch keinen Sommer; Kaffee und Kuchen).

Diese meines Erachtens theoretisch zwingende Entwicklung wirft erhebliche methodische Probleme für die Forschung auf. Wenn es nicht die strukturelle Irregularität ist, wie ist dann das Erkennen der entsprechenden Einheiten idiomatischer Prägung möglich und wie kann operational ihre Abgrenzung gesichert werden?

\section{Konstruktive Pragmatik}

Ein erneuter qualitativer Umschlag in der theoretischen Diskussion ist beobachtbar ab Beginn der 90er Jahre. Die Diskussion ist zum einen gekennzeichnet durch die Bearbeitung der durch die veränderten Grundannahmen der Pragmatisierungsphase neu entstehenden Probleme. Sie ist zum zweiten gekennzeichnet durch die Konvergenz dieser Probleme mit Fragen und The- 
orien, die in Forschungskontexten außerhalb der Phraseologie neu entstehen und ihr entgegenkommen. Dazu zähle ich vor allem die Kontextualisierungstheorie, die Formulierungs- und Textproduktionsforschung sowie jüngere Entwicklungen in der Grammatik, construction grammar und Grammatikalisierungstheorie.

\subsection{Kontext \& Kontextualisierung}

„Routineformeln sind Ausdrücke, deren Vorkommen eng gebunden ist an Typen rekurrenter sozialer Situationen" definiert Florian Coulmas schon Ende der 70er Jahre (Coulmas 1979, S. 239). Insofern sie der sprachliche Ausdruck des sozial-institutionell geregelten Handelns sind, bestimmt der jeweils objektive situative Kontext innerhalb einer sozialen Struktur den Wert der Formel als Begrüßung, Verabschiedung, Entschuldigung usw. Wenn schon die Formel nicht intern fixiert ist, so scheint wenigstens in ihrer äuBeren Bindung ein objektiviertes Kriterium vorzuliegen. Die Vorstellung von der Bindung des Sprachhandelns durch Kontexte mit ihren objektiven Parametern wie Institution, Handlungsrolle, Themen usw. ist ein fester Bestandteil der Pragmatik der späten 70er und der 80er Jahre. Das Problem dieser Sichtweise ist: Kontexte und Kontextparameter sind nicht einfach objektiv gegeben. Soziologisch ist etwa die Handlungsrolle nicht nur Ergebnis eines role taking, sondern ebenso eines role-making, eines konstruktiven, situationsdefinierenden Vorgangs. Der Kontext, in dem sich zwei alte Freunde und Berufskollegen etwa in einer Arztpraxis begegnen, ist nur bei sehr oberflächlicher Betrachtung objektiv gegeben. Tatsächlich wird er erst durch die Handelnden erzeugt: Sie können etwa als alte Freunde ein vor langem gemeinsam bestandenes Abenteuer thematisieren; genauso gut können sie aber auch als Berufskollegen ein Fachthema aufwerfen und eine für die Außenstehenden völlig unverständliche, erhitzte Diskussion dazu führen oder aber - um dies nicht zu vergessen - sie können sich als Patienten in einem bekannten konversationellen Muster ihr Leid klagen. Alles dies ist möglich, und was in dem beschriebenen äußeren objektiven Rahmen dieses Wartezimmers geschieht, ist jeweils sehr verschieden. Wenn der Kontext das Handeln bestimmen soll, tatsächlich aber das Handeln notwendig ist, um die Kontextparameter festzulegen, ist jede auf den Kontext als Explanans rekurrierende Theorie zirkulär. Wie kommt die pragmatische Perspektive aus diesem Zirkel heraus? Die Antwort ist einfach und sie ist einer der Gründe dafür, dass ich im Blick auf die dritte Phase von "konstruktiver Pragmatik“ spreche: Die Beteiligten selbst konstruieren durch ihr Sprechen die für die Verständigung relevanten Kontexte. Weil das Argument vielfach im Sinne einer unterstellten Beliebigkeit der sozial-konstruktiven Prozesse missverstanden wird, möchte ich es explizieren:

Es geht mir mit dieser Feststellung nicht darum, die institutionale Rahmung des Handelns, wie sie etwa die funktionale Pragmatik betont, als theo- 
retisches Konzept über Bord zu werfen. Das Gegenteil ist der Fall. Institutionale Kontexte sind jedoch nicht selbstevident, sie werden im Sinne Max Webers kulturell durch ein wechselseitig sinnhaft aneinander orientiertes Verhalten hervorgebracht und müssen als solche von den Handelnden ständig bestätigt, reproduziert und neu erzeugt werden. Der institutionale wie auch der thematische kontextuale Rahmen der Verständigung ist uno actu konfundiert im kontexterzeugenden semiotischen Handeln der Akteure. Die Leistung der Subjekte in diesem Prozess ist theoretisch meines Erachtens unhintergehbar, und nur der Rückgriff auf deren originären Kontextualisierungsbeitrag kann die mit der sprachlichen Ausdrucksbildung auch verbundene soziale Ordnungsleistung erklären. Jede konkrete Situation ist durch das Handeln grundsätzlich mehrfach bestimmbar und daher pragmatisch zunächst polyvalent. Für die Verständigung muss deshalb in aller Regel ein Kontext zunächst hergestellt, er muss kontextualisiert werden.

Damit ist die Kontextualisierungstheorie von Fillmore (1976), Gumperz (1982), Auer $(1986,1992)$ und anderen angesprochen, die für die Klärung der einschlägigen Probleme in der Phraseologie eine produktive Rolle spielen kann. Peter Auer resümiert den zeichentheoretischen Kern des Ansatzes, wenn er schreibt:

\footnotetext{
„Daraus wird deutlich, dass Kontextualisierung eine zeichenhafte Beziehung zwischen einem (Oberflächen)Merkmal sprachlicher oder nichtsprachlicher Handlungen auf der Ausdrucksebene und einer komplexen semantischen Struktur etabliert, die von der des gewohnten sprachlichen Zeichens mit signifiant und signifié beträchtlich abweicht; während das traditionelle sprachliche Zeichen eine Bedeutungsbeziehung etabliert, indiziert der Kontextualisierungshinweis ein Schema." (Auer 1986, S. 25)
}

Die Kontextualisierungstheorie macht das pars-pro-toto-Prinzip (pars = Oberflächenmerkmal, totum $=$ Schema) zum kommunikationssemantischen Grundprinzip. Grundlegende Überlegungen dazu finden sich bereits bei George Herbert Mead (1934/1973) und Gregory Bateson (1972/1992), der schreibt: „Man wird feststellen, dass diese Weise über Kommunikation nachzudenken, alle Methoden der Codierung unter die einzige Rubrik des pars pro toto einordnet." (Bateson, S. 534)

Wenn Sie jemanden mit „Hallo“ begrüßen, kann es gut sein, dass er Sie freundlich anlächelt, mit "Hallo" zurückgrüßt und weiterzieht. Wenn Sie ihn allerdings mit den Worten empfangen: „Gut, dass ich Sie treffe!“ ist es unwahrscheinlich, dass er „Hallo“ ruft und sich davonmacht. Der Ausdrucksgebrauch erzeugt kontextuelle Optionen und Obligationen, wenn auch hier nur im Blick auf einen einfachen Parameter wie face-to-face vs. nicht-faceto-face (vgl. zu diesem Beispiel ausführlich Feilke 1996). In gleicher Weise können durch idiomatisch geprägte Ausdrücke - bei voller Kompositionalität und syntaktischer Regelhaftigkeit - Sprecherrollen, Themen, ja ganze 
Diskurse und Diskurs-Mentalitäten der Handelnden kontextualisiert werden. Am Beispiel einer Gebrauchsanalyse des Ausdrucks Wir haben uns köstlich amüsiert in Briefen und Tagebüchern junger Mädchen aus bürgerlichem Hause am Anfang des 19. Jh. hat Angelika Linke entsprechende kulturell hochgradig indikative Kontextualisierungsleistungen belegt (vgl. Linke 1996).

Idiomatische Prägungen binden - kraft ihres ausdrucksseitig distinktiven Signalwerts - semiotisch in der Kompetenz der Sprecher ein reichhaltiges Wissen zu Kontexten und befähigen deshalb die Sprecher dazu, diese Kontexte in der Interaktion gleichsinnig zu erzeugen. Wenn Igor Mel'čuk feststellt: „People don't speak in words, they speak in phrasemes" (1995, S. 169), dann ist dies die linguistische Antwort auf das Kontextualisierungsproblem. Spezifische Kontexte sind ansprechbar durch den - gegenüber dem Wort und gegenüber freien Kombinationen - gesteigerten Distinktionswert von Syntagmen, Kollokationen und Formeln, die pars pro toto - als Figur vor einem Hintergrund - genau diesen Hintergrund semantisch indizieren können.

Wenn ich im Folgenden von Kollokationen spreche, sind damit nicht-phraseologische polylexikale, auf der Ebene von Konstituenten und Konstituentenbeziehungen integrierte Ausdrücke gemeint, wobei Auto- und Synsemantika, zwei und mehr Glieder beteiligt sein können (z. B.: von Anfang an, viel zu tun haben, zwar-aber).

\subsection{Usuelle Rekurrenz und Kollokation}

Im Blick auf das Rekurrenzkriterium, also das Kriterium des wiederholten Gebrauchs, gibt es weit verbreitet ein Missverständnis, das theoretisch von einiger Tragweite ist. Die Wiederholung wird im Sinne der Prägung eines motorischen oder sensorischen Engramms als Ursache der Festigkeit gesehen. Als Folge seiner Frequenz ist der Ausdruck gespeichert und wird als ganzer reproduziert. Es gibt auch in der Fachwissenschaft ungezählte Belege für die Auffassung. Ich halte diese Sicht für die psychologische Reduktion eines nur linguistisch zu lösenden Problems und lehne sie aus folgenden Gründen ab. Wenn idiomatische Prägungen ein Gegenstand der Linguistik sind, dann sind sie als Zeichen zu analysieren, das heißt als relativ motivierte, arbiträre und konventionelle Zuordnungen von spezifischen Ausdrucksmerkmalen und Inhalten i.w.S., ganz gleich ob man sich diese Zuordnung etwa nach dem Saussureschen Modell oder nach dem eben vorgestellten pars-pro-toto-Modell der Kontextualisierungstheorie vorstellt. Für die Qualität und die Leistung eines Zeichens ist es ganz unerheblich, wie häufig es auftritt. Ebenso unerheblich ist, dass es reproduziert und gespeichert ist. Das Kriterium des mehr oder weniger frequenten Gebrauchs oder gar die Kriterien der Reproduziertheit und Gespeichertheit, zu denen die Linguistik kraft Amtes gar nichts sagen kann - und meines Erachtens auch nichts sagen soll- 
te -, trägt zur Qualifizierung in Frage stehender Einheiten als Zeichen nichts bei. Im Bereich der Katalysatortechnik beispielsweise gibt es die Kollokation einen Temperaturbereich durchfahren (Schwalm 1991). Sie ist so selten, dass allgemeinsprachliche Wörterbücher sie nicht verzeichnen. Es gibt ungezählte solcher Kollokationen, die wir noch nie produziert und noch nie gehört haben. Manche haben wir vielleicht nur ein- oder zweimal gehört und noch nie gebraucht. Aber beeinträchtigt das ihren Status als Zeichen? Wohl kaum. Selbst wenn uns der Ausdruck einige tausend Male vorgetragen und wir ihn einige tausendmal produziert hätten, würden wir ihn deshalb noch lange nicht als Zeichen kennen oder gar seinen Gebrauch beherrschen. Entscheidend ist alleine die Kenntnis des Gebrauchszusammenhangs. Hier reicht dann unter Umständen die einmalige Erfahrung des Gebrauchszusammenhangs, um den Zeichenwert der Formulierung zu erkennen.

Im gleichen Sinne, wie die Perspektive einer konstruktiven Pragmatik an die Stelle der Bindung des Ausdrucksgebrauchs an vermeintlich objektive Kontexte die Konstruktion der Kontexte durch den Ausdrucksgebrauch rückt, rückt sie auch an die Stelle des vermeintlich objektiven Faktums einer bloß statistischen Rekurrenz die Zeichenkenntnis der Sprecherinnen und Sprecher, ihre idiomatische Kompetenz. Bloße Rekurrenz ist ein gänzlich unzureichendes Kriterium der Kollokationsanalyse. Entscheidend bleibt letztlich die intersubjektiv zu bestätigende idiomatische Kompetenz der Untersucher selbst, die die Belege zu bewerten haben, ein Punkt auf dem Franz Josef Hausmann mit Recht immer wieder insistiert hat. In methodischer Hinsicht ist rekurrente Kookkurenz von Wörtern deshalb vor allem ein heuristisch wertvolles Hilfsmittel für die Entdeckung potentieller idiomatischer Prägungen - z. B. von Kollokationen.

\subsection{Produktionsidiome und die Folgen für die Theorie}

Die rekurrente Kookkurenz als ein bloß statistisches Datum ist also einerseits kategorial verschieden von der Kollokation als Zeichen, anderseits und darauf möchte ich im Folgenden zu sprechen kommen - werfen die Ergebnisse der korpusstatistischen Analyse neue theoretische Probleme auch für den Zeichenbegriff selbst auf, vor allem dadurch, dass sie das relative empirische Gewicht von zum Teil bekannten Sachverhalten in neuem Licht erscheinen lassen.

Das betrifft sowohl die Phraseologie im engeren Sinne als auch und im Besonderen den Bereich der Kollokationen in seiner Beziehung zur usuellen Kookurrenz. Das konstatierte Defizit bloßer Auszählungsverfahren erweist sich dabei in der Konsequenz als theoretisch produktiv oder, wie Dimitrij Dobrovol'skij pointiert formuliert: „Das Innovative des korpusbasierten $\mathrm{Zu}$ gangs zu Kollokationen resultiert in erster Linie aus seiner semantischen Blindheit" (Dobrovol'skij 2002, S. 2; Ms.). Ein positives Ergebnis dieser semantischen Blindheit ist, dass die Aufmerksamkeit durch die Korpusanaly- 
se verstärkt auf idiomatische Prägungen gelenkt wird, die als reguläre Kombinationen freier lexikalischer und grammatischer Komponenten allein ausdruckseitig festgelegt sind und insofern der nichtstatistischen Analyse fast nicht zugänglich sind. Sie belegen eine versteckte semiotische Usualität, von deren Umfang wir uns bisher kaum ein Bild machen können.

Die Kookkurenzanalyse lenkt den Blick zum einen auf bekannte restringierte Kollokationen wie „schütteres Haar" und bestätigt hierin die Intuition, sie lenkt den Blick aber auch auf die Tatsache, dass typische usuelle Kookkurenzen im Sinne einer Gebrauchsnorm die starke Asymmetrie, wie sie bei der klaren Verteilung von Basis und Kollokator im Modell Hausmanns (1985) vorliegt, gar nicht aufweisen müssen: schütter ist textstatistisch betrachtet, kein typischer Partner von Haar, viel eher kommen zum Beispiel empirisch lang und kurz in Frage, wie Kathrin Steyer (2000, S. 110) zeigt. Aber ist die typische Kookkurenz von lang und Haar eine Kollokation, für die Hausmann, Benson u. a. mit Recht eine arbiträre und konventionelle Kombination voraussetzen? Auf den ersten Blick wird man dies verneinen. Es handelt sich um eine freie Wortverbindung. Es gibt keinerlei wortsemantisch zuschreibbare Kombinationsrestriktion. Aber hier ist stets Vorsicht angebracht. Hochgradig rekurrente Kookurrenzen können rein ausdruckseitige Obligationen verstecken, die den beteiligten Lexemen im Sinne einer klaren Basis-Kollokator-Relation nicht zuschreibbar sind. Irmhild Barz (1996) hat im Blick auf den Unterschied zwischen Kollokationen und Komposita (z. B. dicke Milch vs. Dickmilch) darauf hingewiesen, dass die Kollokation eher charakterisiert, das Kompositum eher kategorisiert. Das Verhältnis von individueller Charakterisierung und Kategorisierung ist ein Kontinuum, das durch unterschiedliche Konstruktionen geprägt sein kann. Ist Er hat Haare, die lang sind synonym zu Er hat lange Haare? Die gröBere lexikalische Kohäsion des Adjektivattributs stärkt nach meinem Dafürhalten die Kategorisierung oder auch Stereotypisierung, die freilich am stärksten im Kompositum ausgeprägt ist: der Langhaarige. Damit wäre die Kookkurrenz von lang und Haare in Verbindung mit der Konstruktion als unmittelbar adnominales Adjektivattribut selbst schon signifikativ. Insofern die attributive Bezeichnung des Stereotyps gegenüber den grammatisch möglichen Paraphrasen arbiträr und konventionell ausgezeichnet ist, hat sie den Charakter eines idiomatisch geprägten Zeichens. Lange Haare wäre die Kollokation zur Bezeichnung des Stereotyps.

Etwas anders liegt das Problem bei dem zu einiger Bekanntheit gelangten Beispiel der Kookkurenz von Zähne und putzen. Auch die Zähne putzen erscheint als freie Kombination. Die Analyse, die ich an dieser Stelle nicht mehr vorführen möchte, belegt aber eindeutig die Arbitrarität und Konventionalität der Kombination. In diesem Fall liegt keine Distinktion gegenüber grammatischen Paraphrasen vor, sondern eine arbiträre und konventionelle Selektion aus dem Verbparadigma. Waschen, säubern, reinigen könnten in Frage kommen, aber in ca. 90 Prozent der Fälle heißt es Zähne putzen. 
Kollokationen: arbiträre Selektion

\begin{tabular}{|l|l|l|l|l|l|l|l|}
\hline waschen & $\begin{array}{l}\text { mit } \\
\text { Wasser }\end{array}$ & $\begin{array}{l}\text { mit } \\
\text { Gerät }\end{array}$ & $\begin{array}{l}\text { von } \\
\text { außen }\end{array}$ & putzen & $\begin{array}{l}\text { mit } \\
\text { Wasser }\end{array}$ & $\begin{array}{l}\text { mit } \\
\text { Ger ät }\end{array}$ & $\begin{array}{l}\text { von } \\
\text { außen }\end{array}$ \\
\hline Haare & + & - & + & Nase & - & + & - \\
\hline Hände & + & - & + & Zähne & + & + & - \\
\hline Ohren & + & $-1+$ & - & Schuhe & - & + & + \\
\hline Füße & + & - & + & Stiefel & - & + & + \\
\hline Gesicht & + & - & + & Brille & - & + & + \\
\hline Auto & + & + & + & Zimmer & + & + & $+1-$ \\
\hline Wäsche & + & - & + & Treppe & + & + & + \\
\hline Hemden & + & - & + & Haus & + & + & - \\
\hline Strümpfe & + & - & + & Fenster & + & + & + \\
\hline Gläser & + & - & - & Silber & - & + & + \\
\hline Geschirr & + & + & + & Türklinke & - & + & + \\
\hline Teller & + & + & + & Pferde & - & + & + \\
\hline Gold & + & + & + & Gemüse & - & + & $+1-$ \\
\hline
\end{tabular}

aus: Feilke (1998, S. 75)

Makkai hat schon im Jahr 1972 im Blick auf die rein ausdruckseitige Prägung des kompetenten Sprechens von ,idioms of encoding“ gesprochen, Produktionsidiomen also, die unbeschadet semantischer Durchsichtigkeit und Grammatizität die Selektion und Kombination konventionell strukturieren. Benson (1990) weist anhand der Unterschiede zwischen britischem und amerikanischem Englisch auf entsprechende Produktionsidiome hin. Die erste Variante ist - so Benson - jeweils die britische, die zweite die amerikanische: Our firm made/did a deal with them; The swimmer had/got a cramp; These decisions are to be made/taken rapidly; The children usually set/lay the table (Benson 1990, cit. nach Smadja 1993, S. 145). Sylviane Granger (1998, S. 153) belegt in einer empirischen Untersuchung die Schwierigkeiten von Fremdsprachenlernern mit Produktionsidiomen: Auf das Signalwort highly respondieren native speakers des Englischen (Studierende) zu 80 Prozent mit highly significant, während EFL-Studierende lediglich zu 33 Prozent entsprechend respondieren. Die native speakers respondierten insgesamt nur mit vier Varianten, die EFL-Studierenden mit sieben Varianten.

Die Formulierungstheorie und empirische Textproduktionsforschung, aber auch Bereiche der Grammatiktheorie, stoßen im Übergang zu den 90er Jahren auf die gleichen Probleme. Kombination und Selektion in natürlichen 
Sprachen sind regelgeleitet und werden zugleich in erheblichem Umfang arbiträr und konventionell strukturiert. Formulierungen sind nicht einfach Anwendungen sprachlicher Zeichen und Regeln. Sie sind immer auch Problemlösungen für den Handlungsbedarf. Jede expressive, darstellende oder appellative Sprachhandlung schafft potentiell auch Verbindlichkeiten hinsichtlich der zukünftigen Ausdrucksmöglichkeiten. Aus diesem Grund konstatiert Gerd Antos zu Beginn der 90er Jahre in seiner Einleitung zu einem Sammelband zur Textproduktionsforschung eine „Rehabilitierung der sprachlichen Oberfläche“ (Autos 1989, S. 43). Das betrifft nicht allein den Bereich der Lexik im herkömmlichen Sinne, zu dem ja die Kollokationen gemeinhin gerechnet werden. Es betrifft gerade auch die Frage, inwieweit grammatische Konstruktionen als ,idioms of encoding“ als Folge bewährter grammatischer Muster der Textproduktion funktionsspezifisch geprägt sind. Der Bereich sogenannter grammatical collocations oder der lexico-grammar i. S. des britischen Kontextualismus umfasst nicht nur lexikalisch positiv bestimmte synsemantische Kollokationen, sondern gerade auch syntaktische Ausdrucksmodelle bzw. Formulierungsmodelle, wie sie Charles Fillmore, Paul Kay und andere verstärkt seit Beginn der 90er Jahre im sogenannten construction grammar approach untersuchen. Der Kern des Konzepts besagt, dass grammatische Konstruktionen als abstrahierte Textbildungsroutinen pragmatisch gewichtet und geprägt sein können. Die Routinen sind in dem Sinne lexikalisiert, dass sie in aller Regel über bestimmte Oberflächeneigenschaften - etwa ein Lexem oder eine morphologische Konstruktion - ansprechbar sind.

Ein schönes Beispiel dafür liefert Christiane v. Stutterheim in ihrer Untersuchung zu Quaestio und Textstruktur (1992) mit Ausdrücken wie den folgenden:

- dann die Regenjacke überziehen

- abschließend das kochende Wasser aufgießen usw.

Das Bildungsmuster ist sofort erkennbar. Die syntaktische Struktur ist hier textfunktionsspezifisch (Instruktion) markiert (vgl. Kohlmann 1992): Person, Tempus und Modus sind bereits implizit kontextuell gesetzt. Zu nennen sind lediglich die Fokuselemente: Das Vollverb rückt ans Satzende und steht damit im „Fokus“ der Topik/Fokusstruktur. Es würde sonst bei synthetischen Verben wie überziehen und aufgießen an zweiter Stelle stehen und weniger beachtet werden. Hier geht es also um kontextuell motivierte und markierte morphosyntaktische und Stellungsmuster. Das Muster ist bekannt und wird für eine kompetente Produktion von Instruktionen vorausgesetzt. Die Motivierung des Musters wird gewissermaßen mit konserviert:

„Hier zeigt sich, dass Strukturen, die durch das syntaktische System einer Sprache als [kontextunabhängige H. F.], unveränderliche Determinanten im Sprachproduktionsprozess wirksam zu sein scheinen, letztlich ihrerseits motiviert sind durch die Einbindung der jeweiligen Form bzw. Struktur in einen spezifischen Kontext." (v. Stutterheim 1992, S. 169) 
Auch dieses Zitat verdeutlicht erneut den Gesichtspunkt einer konstruktiven Pragmatik: Die Leistungs-Einheiten einer idiomatischen Kompetenz werden durch die und in der Textproduktion selbst gewonnen und konstruiert. Aufgegeben wird im construction grammar approach die Vorstellung, lexikalische Einheiten - die dann nur als syntaktisch vollständig subkategorisierte Lexemäquivalente denkbar wären - würden nach bestimmten Regeln in eine syntaktisch-logische Form eingesetzt und dann kompositionell interpretiert. Vielmehr gehen Paul Kay und Charles Fillmore davon aus, dass lexikalisierte grammatische Konstruktionen - etwa die Konjunktion geschweige denn mit ihren obligatorischen slots für den komplexen Satz - zum Ausgangspunkt der Phrasen und Satzbildung werden können (vgl. Fillmore et al. 1988).

An die Stelle einer Einsetztheorie rückt Ray Jackendoff (1995) deshalb eine Erlaubnistheorie. Danach genügt es, wenn die elementaren Einheiten den Beschränkungen der Grammatik entsprechen und aussehen, wie syntaktisch reguläre Wörter, Phrasen, Sätze etc. Ich bleibe in der Metaphorik Jackendoffs, der sagt: Die autonome Syntax schaut die Konstruktionen dann nicht wirklich an, so wenig wie sie auch die metrische oder silbenphonologische Struktur von Wörtern anschaut oder sieht, solange diese bestimmten Beschränkungen genügt. Für das Funktionieren idiomatisch geprägter Ausdrücke reicht auch die direkte Zuordnung von phonologischer und konzeptueller Struktur.

Nach dieser Konzeption besteht das Lexikon nicht nur aus Lexemen und es wäre auch unsinnig grammatische Konstruktionen und idiomatisch geprägte Ausdrücke als Lexeme behandeln zu wollen. Vielmehr stehen sie als syntaktisch regulär strukturierte und bereits interpretierte Ausdrücke im Lexikon oder, wie Becker (1975) formuliert hat, im „Phrasikon“ der Sprache. Damit ist das Postulat der Redundanzfreiheit des Lexikons nachhaltig in Frage gestellt, was aber der Theorieentwicklung zum Lexikon im Zeichen von Grammatikalisierungs- und Lexikalisierungstheorie entspricht (vgl. Coulmas 1985, Pawley 1986, Schindler 2002).

Mit den zuletzt angesprochenen pragmatisch zentralen Problembereichen bewegt sich die Sprachtheorie wie auch die Phraseologie selbst weit außerhalb der im Zentrum-Peripheriemodell angelegten Prioritäten der Phraseologie. Das vermeintlich Freie in der Sprache ist, wenn auch nicht fest, so doch in erheblicher und bisher nicht ausgemessener Reichweite idiomatisch geprägt. Umgekehrt gilt, und auch das belegt die korpuslinguistische Forschung eindrücklich: Das so genannte phraseologische Zentrum spielt im Gesamtspektrum der idiomatischen Prägung keine zentrale Rolle. Rosamund Moon resümiert ihre jüngste aufwändige Untersuchung zu den Quantitäten unterschiedlicher Gruppen des Spektrums mit der Feststellung: „I do not wish to overstate my case ... However it seems reasonably certain, that most phrasal lexems are indeed infrequent." (Moon 1998, S. 100). Auch 
wenn zu prüfen ist, wie das für verschiedene Sprachen und Korpora aussieht, bleibt der Befund einer quantitativen Nachrangigkeit im Gesamtspektrum.

Und er steht nicht für sich: Der vermeintlich feste Bereich der Idiome und Phraseolexeme ist tatsächlich gar nicht fest. Im Ergebnis ihrer Untersuchung spricht wiederum Rosamund Moon (1998) von einer ,überwältigenden Evidenz für das Phänomen der Variabilität und extremen Flexibilität“ (ebd., S. 100). Auch in dieser Hinsicht wirkt die Korpusforschung aufklärend.

Als Ergebnis der hier vorgestellten theoretischen Überlegungen und empirischer Untersuchungen, wie ich sie hier referiert habe, schlage ich vor, das Bild des Spektrums der ,mehr oder weniger festen Wortverbindungen“ neu zu entwerfen, bzw. die bisherige Darstellung des Zentrum-PeripherieModells aufzugeben und an dessen Stelle ein Ebenen-Modell wie das folgende zu setzen:

\section{Ebenen-Modelle}

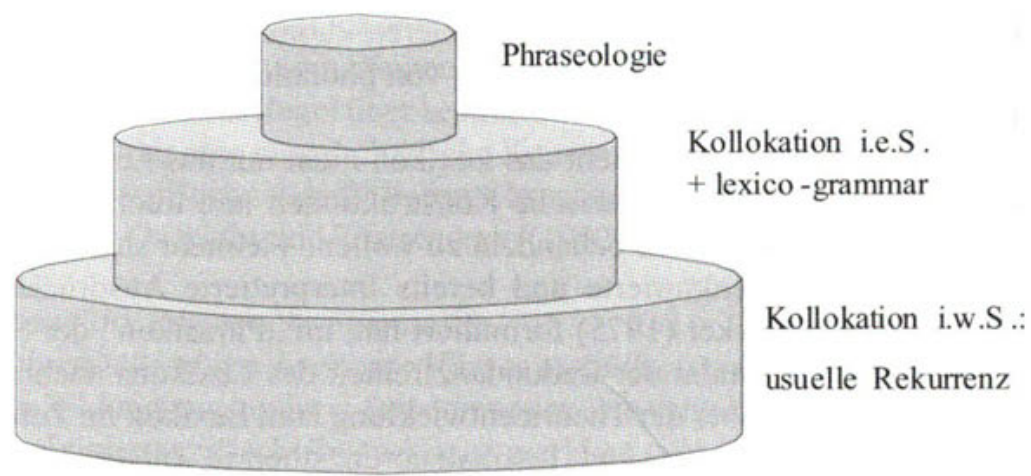

z.B. Gledhill (2000)

Was im Zentrum-Peripherie-Modell bisher peripher erscheint, wird hier zum Fundament. Zugleich wird damit der Blick umgelenkt von der Konzentration auf irreguläre Formen hin zur Konzentration auf die pragmatisch bestätigten regulären Selektionen und Kombinationen in der Sprache oder genauer: in einer Sprache.

\subsection{Wort und Ausdruck}

Wie passen die Beobachtungen zusammen? Ich möchte mit einem Vorschlag schließen, der sich auf das Verhältnis von Wort und idiomatisch geprägtem Ausdruck bezieht. Statt den idiomatischen Ausdruck nach dem Modell des Wortes, des Lexems also, zu behandeln, wie es in der Phraseologie weithin 
üblich ist, schlage ich vor, Wort und Ausdruck grundbegrifflich zu unterscheiden und diesen Unterschied theoretisch stark zu machen (vgl. auch Feilke 1998, 2003).

Das Wort ist die kleinste syntaktisch feldfähige semantische Einheit. Es ist systemintern durch seine syntaktischen und morphosyntaktischen Eigenschaften maximal auf syntaktische Prozesse beziehbar. Gleichzeitig trägt es mit dazu bei, die semantische Prägung von Wortkombinationen und -folgen im Gebrauch gestalthaft zu verkörpern und ins Lexikon zu überführen. Das ist das Feld des idiomatischen Ausdrucks, das bereits bei der Wortbildung beginnt. Der idiomatische Ausdruck entfaltet sein Potential gerade außerhalb der syntaktischen und semantischen Domäne des Wortes, da er vor allem auch illokutionäre, propositionale und textuelle Ordnungsleistungen des Sprechens fasst.

Wort und idiomatischer Ausdruck haben Zeichenqualität. Dabei sind sie als lexikalische Größen in ihrem Funktionieren in komplementärer Weise aufeinander bezogen: Das Wort - im Ausdruck - sichert die interne Passung des jeweiligen Sprechens zur Syntax hin und positioniert es grammatisch. Der Ausdruck sichert die externe Passung des Sprechens zu den erfolgreichen und bestätigten semantischen Orientierungen der Verwendung hin.

Vor diesem Hintergrund wird auch die enorme Flexibilität des phraseologischen Bereichs i.e.S. verständlich. Zwar ist die syntaktische und semantische Beweglichkeit von Phraseologismen, gemessen an einer lexikographisch idealisierten Standardform, erheblich eingeschränkt. Pragmatisch weit wichtiger aber als die getreue Reproduktion ist es, den Phraseologismus überhaupt syntaktisch und situativ passend unterzubringen. Das leistet die in aller Regel erhaltene morphosyntaktische und syntaktische Beweglichkeit der Wörter im Phraseologismus. Danach wäre nicht, wie wir eigentlich geneigt sind anzunehmen, die formativ-strukturelle und semantische Beschränkung und Irregularität entscheidend, sondern gerade im Gegenteil, die semantische und pragmatische Prägung bei gleichzeitig möglichst flexibler Einsetzbarkeit. In genau diesem entscheidenden Punkt sind die Funktionen von Wort und idiomatischem Ausdruck nicht gleich, sondern pragmatisch komplementär.

\section{Resümee}

Der Beitrag hat die Problemgeschichte der Phraseologie aufgegriffen, um zu zeigen, wie die Entwicklung der Phraseologie selbst als eine sukzessive Lösung und Veränderung sprachtheoretischer Fragestellungen verstanden werden kann. Gerade weil die Phraseologie am Anfang ihrer Entwicklung ein sehr eng umgrenztes Gegenstandsverständnis hat, stößt sie innerhalb der etablierten Denkweise immer wieder auf Probleme, die neue theoretische Anstrengungen verlangen. Dabei kommt es nicht nur quantitativ zu einer Ausdehnung des phraseologischen Gegenstandsbereichs, sondern zu einer 
Veränderung der zugrunde liegenden theoretischen Kategorien selbst, die unter dem Stichwort „Pragmatisierung“ gefasst worden ist. Treibende Kraft dieser Entwicklung ist das Bemühen, die Konstituenz und das Funktionieren all derjenigen Einheiten zu erklären, die die Sprecher pragmatisch als Einheiten kennen und behandeln, bzw. deren Kenntnis für einen idiomatisch kompetenten Sprachgebrauch vorausgesetzt werden muss. Die Quintessenz des Beitrags wird im Folgenden in fünf theoretischen Leitkonzepten zusammengefasst:

1. Der Beitrag plädiert erstens für die theoretische Entkopplung von idiomatischer Prägung einerseits und syntaktisch-semantischer Kompositionalität andererseits. Sprachliche Ausdrücke können zugleich idiomatisch geprägt und syntaktisch-semantisch wohlgeformt sein. Lexikalisiertheit und Grammatikalität können als Eigenschaften sprachlicher Zeichen gleichzeitig gegeben sein. Es ist entsprechend theoretisch und empirisch produktiv, den Begriff konventioneller Zeichenhaftigkeit auch auf strukturell motivierte Einheiten (Phrasen, Sätze) auszudehnen (Mel'čuk, Jackendoff).

2. Der Beitrag plädiert zweitens für die pragmatische Revidierung des bilateralen Zeichenmodells. Idiomatisch geprägte Ausdrücke funktionieren als arbiträr-konventionelle und relativ motivierte Figur-Hintergrund-Zeichen. Der in aller Regel strukturell komplexe Ausdruck (Figur) indiziert ein Schema (Hintergrund). Die in dieser Weise auf idiomatische Prägung gestützte pars-pro-toto-Konstitution von Bedeutung (Kontextualisierungssemantik) ist kommunikationssemantisch prioritär gegenüber kompositioneller Bedeutungskonstitution (Mead, Bateson, Gumperz, Auer).

3. Der Beitrag plädiert drittens für die Anerkennung der Relevanz einer rein ausdruckseitig bestimmten Idiomatik (Produktionsidiome, idioms of encoding) für die Bestimmung der Sprachkompetenz.

4. Der Beitrag plädiert viertens dafür, die Kategorie Ausdruck als eigenständige Ebene des Sprachsystems und als semantisch und pragmatisch komplementäres Konzept zum syntaktischen Wort zu etablieren.

5. Schließlich ergibt sich aus der Argumentation fünftens das Plädoyer für die Ablösung des Zentrum-Peripherie-Modells der Phraseologie durch ein Ebenenmodell idiomatischer Prägung.

\section{Literatur}

Antos, Gerd (1987): Grußworte in Festschriften als ,institutionale Rituale‘. In: Zeitschrift für Literaturwissenschaft und Linguistik 65, S. 9-40.

Antos, Gerd/Krings, Hans P. (Hg.) (1989): Textproduktion. Ein interdisziplinärer Forschungsüberblick. Tübingen.

Antos, Gerd (1989): Textproduktion: Ein einführender Überblick. In: Antos, G./Krings, H.P. (Hg.): Textproduktion. Tübingen. S. 5-57. 
Auer, Peter (1986): Kontextualisierung. In: Studium Linguistik 19, S. 22-48.

Auer, Peter (1992): Introduction: John Gumperz' approach to contextualization. In: Auer/ di Luzio (eds.) (1992), S. 1-37.

Auer, Peter/di Luzio, Aldo (eds.) (1992): The contextualization of language. Amsterdam/ Philadelphia.

Barthes, Roland (1970): Mythen des Alltags. Frankfurt a. M.

Barz, Irmhild (1996): Komposition und Kollokation. Überlegungen zur Lexikalisierung von Nominalkomposita. In: Clemens Knobloch/Burkhard Schaeder (Hg.): Nomination - fachsprachlich und gemeinsprachlich. Opladen. S. 111-130.

Bateson, Gregory (1972/1992): Ökologie des Geistes. 4. Auflg., Frankfurt a. M.

Becker, J.D. (1975): The phrasal lexicon. In: Schank, R./Nash-Webber, B. L. (eds.): Theoretical issues in natural language processing. Cambridge Mass. S. 70-74.

Benson, M. (1990): Collocations and General Purpose Dictionaries. In: International Journal of Lexicography, 3(1), S. 23-35.

Bloomfield, Leonhard (1933): Language. New York.

Bolinger, Dwight (1976): Meaning and memory. In: Forum Linguisticum 1/1, S. 1-14.

Burger, Harald (1973): Idiomatik des Deutschen. Tübingen.

Burger, Harald (1998): Phraseologie. Eine Einführung am Beispiel des Deutschen. Berlin.

Burger, Harald/Buhofer, Annelies/Sialm, Ambros (1982): Handbuch der Phraseologie. Berlin/New York.

Coulmas, Florian (1979): On the sociolinguistic relevance of routine formulae. In: Journal of Pragmatics 3, S. 239-266.

Coulmas, Florian (1981a): Routine im Gespräch. Zur pragmatischen Fundierung der Idiomatik. Wiesbaden.

Coulmas, Florian (1981b): Idiomatizität: Zur Universalität des Idiosynkratischen. In: Linguistische Berichte 72, S. 27-50.

Coulmas, Florian (1985): Lexikalisierung von Syntagmen. In: Handbuch der Lexikologie. Hg. v. Schwarze, C. und Wunderlich, D. Königsstein/Ts. S. 250-268.

Cowie, Andrew P. (ed.) (1998): Phraseology. Theory, Analysis, and Applications. Oxford.

Dobrovol'skij, Dimitrij (1995): Kognitive Aspekte der Idiom-Semantik. Studien zum Thesaurus deutscher Idiome. Tübingen.

Dobrovol'skij, Dimitrij (2002): Restricted Collocations in the „Russian-German Parallel Corpus" (Ms.).

Feilke, Helmuth (1994): Common sense-Kompetenz. Überlegungen zu einer Theorie des ,sympathischen' und ,natürlichen' Meinens und Verstehens. Frankfurt a. M.

Feilke, Helmuth (1996): Sprache als soziale Gestalt. Ausdruck, Prägung und die Ordnung der sprachlichen Typik. Frankfurt a. M.

Feilke, Helmuth (1998): Idiomatische Prägung. In: Barz, Irmhild/Öhlschläger, Günther (Hg.): Zwischen Grammatik und Lexikon. Tübingen. S. 69-81.

Feilke, Helmuth (2003): Textroutine, Textsemantik und sprachliches Wissen. In: Linke, A./ Ortner, H.P./Portmann, P. (Hg.): Sprache und mehr. Tübingen.

Fillmore, Charles J. (1976): Pragmatics and the description of discourse. In: Schmidt, S. J. (Hg.): Pragmatik/Pragmatics II. Grundlegung einer expliziten Pragmatik. München. S. 83-104.

Fillmore, Charles J. (1978): On the organization of semantic information in the lexicon. In: Parasession on the lexicon. [Chicago Linguistic Society] Chicago. S. 148-173.

Fillmore, Charles J. (1979): Innocence: A second idealization for linguistics. In: Berkeley Linguistics Society 5, S. 63-76.

Fillmore, Charles J. (1989): Grammatical construction theory and the familiar dichoto- 
mies. In: Rainer, Dietrich/Carl F. Graumann (eds.): Language processing in social context. Amsterdam/New York/Oxford/Tokyo. S. 17 - 38.

Fillmore, Charles J./Kay, Paul/O'Connor, Mary Catherine (1988): Regularity and idiomaticity in grammatical constructions. The case of ,let alone'. In: Language 64/3, S. 501-538.

Fillmore, Charles (1976): Pragmatics and the description of discourse. In: Pragmatik/ Pragmatics II. Zur Grundlegung einer expliziten Pragmatik. Hg. v. S. J. Schmidt. München. S. 83-104.

Fleischer, Wolfgang (1982): Phraseologie der deutschen Gegenwartssprache. Leipzig.

Gledhill, C. J. (2000): Collocations in Science Writing. Tübingen.

Gläser, Rosemarie (1986): Phraseologie der englischen Sprache. Leipzig/Tübingen.

Gläser, Rosemarie (1988): The grading of idiomaticity as a presupposition for a taxonomy of idioms. In: Hüllen, W./Schulze, R. (Hg.): Understanding the lexicon. Tübingen. S. 264-277.

Granger, Sylviane (1998): Prefabricated Patterns in Advanced EFL Writing: Collocations and Formulae. In: Cowie, A.P. (Ed.): Phraseology. Theory, Analysis, and Applications. Oxford. S. 145-160.

Gréciano, Gertrud (1983): Forschungsbericht: Forschungen zur Phraseologie. In: Zeitschrift für germanistische Linguistik 11, S. 232-243.

Grzybek, Peter (1984a): Überlegungen zur semiotischen Sprichwortforschung. In: Kodikas/Code 7, H3/4, S. 215-249.

Grzybek, Peter (1984b): Zur Psychosemiotik des Sprichworts. In: Kodikas/Code 7, H3/4, S. $409-432$.

Gülich, Elisabeth (1997): Routineformeln und Formulierungsroutinen. Ein Beitrag zur Beschreibung formelhafter Texte. In: Wimmer, R./Berens, F. J. (Hg.): Wortbildung und Phraseologie. Tübingen. S. 131-176.

Gumperz, John J. (1982). Discourse strategies. Cambridge.

Hausmann, Franz Josef (1984): Wortschatzlernen ist Kollokationslernen. Zum Lehren und Lernen französischer Wortverbindungen. In: Praxis des neusprachlichen Unterrichts 31, S. $395-406$.

Hausmann, Franz Josef (1985): Kollokationen im deutschen Wörterbuch. Ein Beitrag zur Theorie des lexikographischen Beispiels. In: Bergenholtz, H./Mugdan, J. (Hg.): Lexikographie und Grammatik. S. 118-129.

Hausmann, Franz Josef (1993): Ist der deutsche Wortschatz lernbar? In: Info Daf 20/5, S. $471-485$.

Hessky, Regina (1987): Phraseologie. Linguistische Grundfragen und kontrastives Modell deutsch-ungarisch. Tübingen.

Hjelmslev, L. (1943/1974): Prolegomena zu einer Sprachtheorie. München.

Hockett, C. F. (1956): Idiom formation. In: Halle, M. et al. (eds.): For Roman Jakobson. Essays on the occasion of his sixtieth birthday. The Hague. S. 222-229.

Jackendoff, Ray (1995): The Boundaries of the Lexicon. In: Everaerts, M. et al. (eds.): Idioms: Structural and Psychological Perspectives. Hillsdale. S. 133-165.

Kay, Paul/Fillmore, Charles (1996): Grammatical Constructions and Linguistic Generalizations: The What's $\mathrm{X}$ doing Y? Construction. Dept. of Linguistics, University of California Berkeley. (Ms.).

Kohlmann, Ute (1992): Textstruktur und sprachliche Form in Instruktionstexten. In: Krings, Hans Peter/Antos, Gerd (Hg.): Textproduktion. Neue Wege der Forschung. Trier. S. 173-192.

Lambrecht, Knud (1984): Formulaicity, frame semantics and pragmatics in german binominal expressions. In: Language 60/4, S. 753-796. 
Linke, Angelika (1996): Sprachkultur und Bürgertum. Zur Mentalitätsgeschichte des 19. Jahrhunderts. Stuttgart/Weimar.

Lüger, Heinz Helmut (1980): Formen rituellen Sprachgebrauchs. In: Deutsche Sprache 8, S. $21-40$.

Lüger, Heinz Helmut (1983): Some aspects of ritual communication. In: Journal of Pragmatics 7, S. 695-711.

Maas, Utz (1985): Konnotation. In: Januschek, F. (Hg.): Politische Sprachwissenschaft. Zur Analyse von Sprache als kultureller Praxis. Opladen. S. 71-97.

Makkai, A. (1972): Idiom structure in english. The Hague.

Mead, George Herbert (1934/1973): Geist, Identität und Gesellschaft. Aus der Sicht des Sozialbehaviorismus. Frankfurt a.M.

Mel'čuk, Igor (1998): Collocations and Lexical Functions. In: Cowie, E. P. (ed.) (1998), S. $23-53$.

Moon, Rosamund (1998): Frequencies and Forms of Phrasal Lexemes in English. In: Cowie, A.P. (ed.) (1998), S. 79-100.

Moon, Rosamund (1998): Fixed Expressions and Idioms in English. A Corpus-Based Approach. (Oxford Studies in Lexicography and Lexicology.) Oxford.

Müller, Gereon (1997): Beschränkungen für Binominalbildungen im Deutschen. In: Zeitschrift für Sprachwissenschaft 16, 1/2, S. 5-51

Pawley, Andrew (1986): Lexicalisation. In: Tannen, D./Atlatis, J. E. (eds.): Language and Linguistics: The interdependence of theory, data and application. Georgetown University Round Table on Languages and Linguistics 1985 [GURT]. Washington DC.S. $98-120$

Pilz, K.D. (1978): Phraseologie. Versuch einer interdisziplinären Abgrenzung, Begriffsbestimmung und Systematisierung. Göppingen.

Pilz, K. D. (1981): Phraseologie: Redensartenforschung. Stuttgart.

Rothkegel, Annely (1994): Kollokationsbildung und Textbildung. In: Sandig, B. (Hg.): Europhras 92. Tendenzen der Phraseologieforschung. Bochum. S. 499-525.

Schemann, Hans (1987): Was heißt ,Fixiertheit' von phraseologischen oder idiomatischen Ausdrücken? In Korhonen, J. (Hg.): Beiträge zur allgemeinen und germanistischen Phraseologieforschung. Oulu. S. 22-36.

Schemann, Hans (1989): Das phraseologische Wörterbuch. In: Hausmann, F. J./ Reichmann, O./Wiegand, H. E./Zgusta, L. (eds.): Wörterbücher. Ein internationales Handbuch zur Lexikographie. Bd.1. [Reihe Handbücher zur Sprach- und Kommunikationswissenschaft]. Berlin/New York. S. 1019-1032.

Schindler, Wolfgang (1996): Phraseologismen und phraseologische Bindungsebenen. Eine Untersuchung zum Gegenstandsbereich der Phraseologie und zu kollokationeller, semantischer lexematischer, syntaktischer und pragmatischer Bindung. (Habilitationsschrift) Institut für Deutsche Philologie, Universität München.

Schindler, Wolfgang (2002): Lexik, Lexikon, Wortschatz: Probleme der Abgrenzung. In: Lexikologie - Lexicology. Ein internationales Handbuch zur Natur und Struktur von Wörtern und Wortschätzen. Bd.1. Berlin/New York. S. 34-44

Schwalm, Gisela (1991): Zum Problem der Kollokation in Gemein- und Fachsprache im Kontext der Übersetzung. In: TextContext 6, S. 163-182.

Smadja, Frank (1993): Retrieving Collocations from text: Xtract. In: Computational Linguistics $19(1)$, S. 143-177

Stein, Stephan (1995): Formelhafte Sprache. Untersuchungen zu ihren pragmatischen und kognitiven Funktionen im gegenwärtigen Deutsch. Frankfurt a. M. et al.

Steyer, Katrin (2000): Usuelle Wortverbindungen des Deutschen. In: Deutsche Sprache 28, S. $101-125$. 
Steyer, Katrin (2002): Wenn der Schwanz mit dem Hund wedelt. Zum linguistischen Erklärungspotenzial der korpusbasierten Kookurenzanalyse. In: Haß-Zumkehr, U./ Kallmeyer, W./Zifonun, G. (Hg.): Ansichten der deutschen Sprache. FS für Gerhard Stickel zum 65. Geburtstag. Tübingen. S. 215-236.

Thun, Harald (1978): Probleme der Phraseologie. Untersuchungen zur wiederholten Rede mit Beispielen aus dem Französischen, Italienischen, Spanischen und Rumänischen. Tübingen.

v. Stutterheim, Christiane (1992): Quaestio und Textstruktur. In: Krings, H. P./Antos, Gerd (Hg.): Textproduktion. Neue Wege der Forschung. Trier. S. 159-171.

Weinreich, Uriel (1969/1980): Problems in the analysis of idioms. In: ders.: On semantics. Ed. by Labov, W. and B. S. Weinreich. University of Pennsylvania Press. S. 208-264. 